\title{
Real-Time Traffic-Differentiated QoS Routing for Wireless Sensor Networks
}

\author{
Shiva Prakash T \\ University Visvesvaraya College of Engineering \\ K R Circle, Bangalore-560 001.
}

\author{
Raja K B \\ University Visvesvaraya College of Engineering \\ K R Circle, Bangalore-560 001.
}

\author{
Venugopal K R \\ University Visvesvaraya College of Engineering \\ K R Circle, Bangalore-560 001.
}

\begin{abstract}
Wireless sensor networks (WSNs) emerge as underlying infrastructures for new classes of large scale networked embedded systems. However, WSNs system designers must fulfill the Qualityof-Service (QoS) requirements imposed by the applications (and users). Very harsh and dynamic physical environments and extremely limited resources are major obstacles for satisfying QoS metrics such as reliability, timeliness, and system lifetime. The limited communication range of WSN nodes, link asymmetry, and the characteristics of the physical environment lead to a major source of QoS degradation in WSNs. This paper proposes a Real-Time Traffic-Differentiated Routing protocol for Wireless Sensor Networks (WSNs). It targets WSN applications having different types of data traffic with several priorities. The protocol achieves to increase packet reception ratio and reduce end-to-end delay while considering multi-queue priority policy, two-hop neighborhood information, link reliability and power efficiency. The protocol is modular and utilizes memory and computational effective methods for estimating the link metrics. Numerical results show that the proposed protocol is a feasible solution to addresses QoS service differentiation for traffic with different priorities.
\end{abstract}

\section{Keywords:}

Wireless Sensor Networks(WSNs), quality-of-service(QoS), twohop neighbors, traffic-differentiation, Packet Reception Ratio(PRR), end-to-end delay.

\section{INTRODUCTION}

Wireless Sensor Networks (WSN) form a network of small and low-powered micro sensing devices denoted as motes [13] that can sense the environment and communicate the information gathered from the monitored field through wireless links; the data is forwarded via multiple hops to a sink.

WSNs have a wide variety of applications in military, industry, environment monitoring and health care. WSNs operate unattended in harsh environments, such as border protection and battlefield reconnaissance hence help to minimize the risk to human life. WSNs are used extensively in the industry for factory automation, process control, real-time monitoring of machines, detection of radiation and leakages and remote monitoring of contaminated areas, aid in detecting possible system deterioration and to initiate precautionary maintenance routine before total system breakdown. WSNs are being rapidly deployed in patient health monitoring in a hospital environment, where different health parameters are obtained and forwarded to health care servers accessible by medical staff and surgical implants of sensors can also help monitor the health of patients. These WSNs have diverse data traffic with different quality of service $(\mathrm{QoS})$ requirements.

The QoS requirements include timeliness, high reliability, availability and integrity. Various performance metrics that can be used to justify the quality of service include, packet reception ratio (PRR), defined as the probability of successful delivery should be maximized. The end-to-end delay which is influenced by the queuing delay at the intermediate nodes and the number of hops traversed by the data flows of the session from the source to the receiver should be minimum. Therefore, providing corresponding traffic differentiation QoS in such scenarios pose to be a great challenge. Our proposed protocol is motivated primarily by the deficiencies of the previous works (explained in the Section 2) and aims to provide better Quality of Service.

This paper explores the idea of incorporating QoS parameters in making routing decisions the protocol proposes the following features.

(1) Data Traffic is split into regular traffic with no specific QoS requirement, reliability-responsive traffic: which should be transmitted without loss but can tolerate some delay, delayresponsive traffic: which should be delivered within a deadline but may tolerate moderate packet loss and critical traffic: which has high significance and demanding both high reliability and short delay.

(2) Link reliability is considered while choosing the next router, this selects paths which have higher probability of successful delivery.

(3) Routing decision is based on two-hop neighborhood information and dynamic velocity that can be modified according to the required deadline, this results in significant reduction in end-to-end PRR. 
Table 1. Our results and comparison with previous results for Differentiated QoS routing in Wireless Sensor Networks.

\begin{tabular}{|c|c|c|c|c|c|c|}
\hline $\begin{array}{l}\text { Related } \\
\text { Work }\end{array}$ & $\begin{array}{l}\text { Protocol } \\
\text { Name }\end{array}$ & $\begin{array}{l}\text { Considered } \\
\text { Metrics }\end{array}$ & $\begin{array}{l}\text { Estimation } \\
\text { Method }\end{array}$ & $\begin{array}{l}\text { Traffic } \\
\text { Differentiation }\end{array}$ & Duplication & Performance \\
\hline $\begin{array}{l}\text { E. Felemban } \\
\text { et al., 12 }\end{array}$ & $\begin{array}{l}\text { MMSPEED } \\
\text { (Multi-path } \\
\text { and Multi-SPEED } \\
\text { Routing Protocol) }\end{array}$ & $\begin{array}{l}\text { one-hop delay, } \\
\text { link reliability } \\
\text { and residual } \\
\text { energy. }\end{array}$ & EWMA & $\begin{array}{l}\text { Delay } \\
\text { requirement }\end{array}$ & $\begin{array}{l}\text { Towards } \\
\text { the same } \\
\text { sink }\end{array}$ & $\begin{array}{l}\text { Provides service } \\
\text { differentiation and } \\
\text { probabilistic QoS guarantee } \\
\text { in the timeliness and } \\
\text { reliability domains. }\end{array}$ \\
\hline $\begin{array}{l}\text { M.M. } \\
\text { Or-Rashid } \\
\text { et al., } \\
{[22}\end{array}$ & $\begin{array}{l}\text { DARA (Data } \\
\text { Aggregate } \\
\text { Routing } \\
\text { Algorithm) }\end{array}$ & $\begin{array}{l}\text { one-hop delay } \\
\text { and transmission } \\
\text { power. }\end{array}$ & Variance-based & $\begin{array}{l}\text { Critical } \\
\text { Traffic and } \\
\text { Non-Critical }\end{array}$ & $\begin{array}{l}\text { Towards } \\
\text { different } \\
\text { sinks } \\
\text { Traffic }\end{array}$ & $\begin{array}{l}\text { The protocol considers } \\
\text { reliability, delay, and residual } \\
\text { energy in the routing } \\
\text { metric, only queuing } \\
\text { time is considered. }\end{array}$ \\
\hline $\begin{array}{l}\text { Y. Li } \\
\text { et al., 34] }\end{array}$ & $\begin{array}{l}\text { THVR (Two-Hop } \\
\text { Velocity Based } \\
\text { Routing Protocol) }\end{array}$ & $\begin{array}{l}\text { two-hop delay } \\
\text { and residual } \\
\text { energy. }\end{array}$ & WMEWMA & No & No & $\begin{array}{l}\text { Routing Decision is made } \\
\text { based on two-hop velocity } \\
\text { integrated with energy } \\
\text { balancing mechanism which } \\
\text { achieves lower E2E delay. }\end{array}$ \\
\hline $\begin{array}{l}\text { D. Djenouri } \\
\text { et al.,11 }\end{array}$ & $\begin{array}{l}\text { LOCALMOR } \\
\text { (Localized } \\
\text { Multi-objectives } \\
\text { Routing) }\end{array}$ & $\begin{array}{l}\text { one-hop delay } \\
\text { link reliability } \\
\text { residual energy } \\
\text { and transmission } \\
\text { power. }\end{array}$ & $\begin{array}{l}\text { EWMA and } \\
\text { WMEWMA }\end{array}$ & $\begin{array}{l}\text { Regular, } \\
\text { Reliability } \\
\text {-sensitive,Delay, } \\
\text {-sensitive and } \\
\text { Critical Traffic }\end{array}$ & $\begin{array}{l}\text { Towards } \\
\text { different } \\
\text { sinks }\end{array}$ & $\begin{array}{l}\text { Traffic classified into several } \\
\text { categories according to the } \\
\text { required QoS, differentiation } \\
\text { of both delay and reliability. } \\
\text { is achieved. }\end{array}$ \\
\hline This paper & $\begin{array}{l}\text { TDTHR } \\
\text { (Traffic } \\
\text {-Differentiated } \\
\text { Two-Hop } \\
\text { Routing) }\end{array}$ & $\begin{array}{l}\text { two-hop delay, } \\
\text { link reliability } \\
\text { residual energy } \\
\text { and transmission } \\
\text { power. }\end{array}$ & $\begin{array}{l}\text { EWMA and } \\
\text { WMEWMA }\end{array}$ & $\begin{array}{l}\text { Regular, } \\
\text { Reliability } \\
\text {-Responsive,Delay, } \\
\text {-Responsive and } \\
\text { Critical Traffic }\end{array}$ & $\begin{array}{l}\text { Towards } \\
\text { different } \\
\text { sinks }\end{array}$ & $\begin{array}{l}\text { The protocol considers } \\
\text { multi-queue priority policy, } \\
\text { two-hop dynamic velocity } \\
\text { assignment policy, link } \\
\text { reliability and power } \\
\text { efficiency. It improves the } \\
\text { PRR and reduces } \\
\text { end-to-end delay. }\end{array}$ \\
\hline
\end{tabular}

(4) Choosing nodes with higher residual energy and minimum trasmission power, balances the load among nodes and results in prolonged lifetime of the network.

We test the performance of our proposed approaches by implementing our algorithms using $n s-2$ simulator. Our results demonstrates the performance and benefits of RTTDR over earlier algorithms. The rest of the paper is organized as follows: Section 2 gives a review of related works. Section 3 and Section 4 detail the studied problem, network model, notations, assumptions and proposed routing algorithm. Section 5 is devoted to the simulation and evaluation of the algorithm. Conclusions are presented in Section 6 .

\section{RELATED WORK}

Stateless routing protocols which do not maintain per-route state is a favorable approach for WSNs. The idea of stateless routing is to use location information available to a node locally for routing, i.e., the location of its own and that of its one-hop neighbors without the knowledge about the entire network. These protocols scale well in terms of routing overhead because the tracked routing information does not grow with the network size or the number of active sinks. Parameters like distance to sink, energy efficiency and data aggregation, need to be considered to select the next router among the one-hop neighbors.

SPEED (Stateless Protocol for End-to-End Delay) [31] is a well known stateless routing protocol for real-time communication in sensor networks. It is based on geometric routing protocols such as greedy forwarding GPSR (Greedy Perimeter State Routing) [6][25]. It uses non-deterministic forwarding to balance each flow among multiple concurrent routes. SPEED combines Medium Access Control (MAC) and network layer mechanism to maintain a uniform speed across the network, such that the delay a packet experiences is directly proportional to its distance to the sink. At the MAC layer, a single hop relay speed is maintained by controlling the drop/relay action in a neighbor feedback loop. Geographic forwarding is used to route data to its destination selecting the next hop as a neighbor from the set of those with a relay speed higher that the desired speed. A back pressure re-routing mechanism is employed to re-route traffic around congested areas if necessary. Lu et al., [7] describe a packet scheduling policy, called Velocity Monotonic Scheduling, which inherently accounts for both time and distance constraints. Sequential Assignment Routing (SAR) [16] is the first routing protocol for sensor networks that creates multiple trees routed from one-hop neighbors of the sink by taking into consideration both energy resources, QoS metric on each path and priority level of each packet. However, the protocol suffers from the overhead of maintaining the tables and states at each sensor node especially when the number of nodes is large.

MMSPEED (Multi-path and Multi-SPEED Routing Protocol) [12] is an extension of SPEED that focuses on differentiated QoS options for real-time applications with multiple different deadlines. It provides differentiated QoS options both in timeliness domain and the reliability domain. For timeliness, multiple QoS levels are supported by providing multiple data delivery speed options. For reliability, multiple reliability requirements are supported by probabilistic multi-path forwarding. The protocol provides end-to-end QoS provisioning by employing localized geographic forwarding using immediate neighbor information without end-to-end path dis- 
covery and maintenance. It utilizes dynamic compensation which compensates for inaccuracy of local decision as a packet travels towards its destination. The protocol adapts to network dynamics. MMSPEED does not include energy metric during QoS route selection. Chipera et al., [24] (RPAR:Real-Time Power Aware Routing) have proposed another variant of SPEED. Where a node will change its transmission power by the progress towards destination and packet's slack time in order to meet the required velocity; they have not considered residual energy and reliability.

DARA [22] considers reliability, delay, and residual energy in the routing metric, and defines two kinds of packets: critical and noncritical packets. The same weighted metric is used for both types of packets, where the only difference is that a set of candidates reached with a higher transmission power is considered to route critical packets. For delay estimation, the authors use queuing theory and suggest a method that, in practice, needs huge amount of sample storages.

Mahapatra et al., [3] assign an urgency factor to every packet depending on the residual distance and time the packet neesds to travel, and determines the distance the packet needs to be forwarded closer to the destination to meet its deadline. Multi-path routing is performed only at the source node for increasing reliability. Some routing protocols with congestion awareness have been proposed in [10]|35]. Other geographic routing protocols such as [33][32]|27||8] deal only with energy efficiency and transmission power in determining the next router. Seada et al., [15] proposed the PRR (Packet Reception Rate) $\times$ Distance greedy forwarding that selects the next forwarding node by multiplying the PRR by the distance to the destination. Recent geographical routing protocols have been proposed, such as DARA (Distributed Aggregate Routing Algorithm) [22], GREES (Geographic Routing with Environmental Energy Supply) [17], DHGR (Dynamic Hybrid Geographical Routing) [20], and EAGFS (Energy Aware Geographical Forwarding Scheme) [29]. They define either the same combined metric (of all the considered QoS metrics) [31], [29], [17], or several services but with respect to only one metric [24], [12].

Sharif et al., [4] presented a new transport layer protocol that prioritizes sensed information based on its nature while simultaneously supporting the data reliability and congestion control features. Rusli et al., [23] propose an analytical framework model based on Markov Chain of OR and M/D/1/K queue to measure its performance in term of end-to-end delay and reliability in WSNs. Koulali et al.,21] propose a hybrid QoS routing protocol for WSNs based on a customized Distributed Genetic Algorithm (DGA) that accounts for delay and energy constraints. Yunbo Wang et al., [36] investigate the end-to-end delay distribution, they develop a comprehensive cross-layer analysis framework, which employs a stochastic queueing model in realistic channel environments. Ehsan et al.,26] propose energy and cross-layer aware routing schemes for multichannel access WSNs that account for radio, MAC contention, and network constraints.

All the above routing protocols are based on one-hop neighborhood information. However, it is expected that multi-hop information can lead to improved performance in many issues including message broadcasting and routing. Spohn et al., [19] propose a localized algorithm for computing two-hop connected dominating set to reduce the number of redundant broadcast transmissions. An analysis in [14] shows that in a network of $n$ nodes of total of $O(n)$ messages are required to obtain 2-hop neighborhood information and each message has $O(\log n)$ bits. Chen et al., [9] study the performance of 1-hop, 2-hop and 3-hop neighborhood information based routing and propose that gain from 2-hop to 3-hop is relatively minimal, while that from 1-hop to 2-hop based routing is significant. $\mathrm{Li}$ et al., [34] have proposed a Two-Hop Velocity Based Routing Protocol (THVR). The routing choice is decided on the two-hop relay velocity and residual energy, an energy efficient packet drop control is included to enhance packet utilization efficiency while keeping low packet deadline miss ratio. Djenouri et al. [11] propose a new localized quality of service (QoS) routing protocol (LOCALMOR) it is based on differentiating QoS requirements according to the data type, which enables to provide several and customized QoS metrics for each traffic category. With each packet, the protocol attempts to fulfill the required data-related QoS metric(s) while considering power efficiency. The protocol proposed in this paper is different from LOCALMOR it considers two-hop transmission delay and queuing delay for selecting the next node.

\section{PROBLEM DEFINITION}

The topology of a wireless sensor network may be described by a graph $G=(N, L)$, where $N$ is the set of nodes and $L$ is the set of links. The objectives are to,

- Maximize the Packet Recepion ratio (PRR).
-Reduce the end-to-end packet delay.
-Improve the energy efficiency (ECPP-Energy Consumed Per
Packet) of the network.

\subsection{Network Model and Assumptions}

In our network model, we assume the following:

- The wireless sensor nodes consists of $N$ sensor nodes and a sink, the sensors are distributed randomly in a field.

- The nodes are aware of their positions through internal global positioning system (GPS), so each sensor has a estimate of its current position.

- The $N$ sensor nodes are powered by a non renewable on board energy source. When this energy supply is exhausted the sensor becomes non-operational. All nodes are supposed to be aware of their residual energy and have the same transmission power range.

- The sensors share the same wireless medium each packet is transmitted as a local broadcast in the neighborhood. The sensors are neighbors if they are in the transmission range of each other and can directly communicate with each other. We assume any MAC protocol, which ensures that among the neighbors in the local broadcast range, only the intended receiver keeps the packet and the other neighbors discard the packet.

—Like all localization techniques, [31][12][28] [18][30] each node needs to be aware of its neighboring nodes current state (ID, position, link reliability, residual energy etc), this is done via HELLO messages.

- Nodes are assumed to be stationary or having low mobility, else additional HELLO messages will be needed to keep the nodes up-to-date about the neighbor nodes.

- In addition, each node sends a second set of HELLO messages to all its neighbors informing them about its one-hop neighbors. Hence, each node is aware of its one-hop and two-hop neighbors and their current state.

- The network density is assumed to be high enough to prevent the void situation. 
Table 2. Notations used in Section 4

\begin{tabular}{ll}
\hline Symbol & \multicolumn{1}{c}{ Definition } \\
\hline$N$ & Set of Nodes in the WSN \\
$D$ & Destination Node \\
$S$ & Source Node \\
$d i s t(x, y)$ & Distance between a node pair $x, y$ \\
$N_{1}(x)$ & Set of one-hop Neighbors of node $x$ \\
$N_{2}(x)$ & Set of two-hop Neighbors of node $x$ \\
$F_{1}^{+p}(x)$ & Set of node $x$ 's one-hop favorable forwarders \\
& providing positive progress towards \\
$F_{2}^{+p}(x, y)$ & the destination D \\
$d t_{x y}$ & Set of node $x$ 's two-hop favorable forwarders \\
& Estimated one-hop transmission delay \\
$d q_{x}$ & between nodes $x$ and $y$ \\
$t_{r e q}$ & Estimated queuing delay at node $x$ \\
$V_{r e q}$ & Time deadline to reach Destination D \\
& Required end-to-end packet delivery \\
$V_{x y}$ & Velocity for deadline $t_{r e q}$ \\
$V_{x y \rightarrow z}$ & Velocity offered by $y \in F_{1}^{+p}(x)$ \\
$S_{r e q}$ & Velocity offered by $y \in F_{2}^{+p}(x, y)$ \\
$T_{a}\left(d i s t(x, y)^{\alpha}\right)$ & Node pairs satisfying $V_{x y \rightarrow z} \geq V_{r e q}$ \\
$E_{y}$ & Transmission power cost from node x to node y \\
$p r r_{x y}$ & Remaining energy of node $y$ \\
$\beta$ & Packet Reception Ratio of link relaying \\
$\gamma$ & node $x$ to node $y$ \\
& Tunable weighting coefficient for $p r r$ estimation \\
& Tunable weighting coefficient for queuing and \\
& transmission delay estimation \\
\hline
\end{tabular}

\section{ALGORITHM}

RTTDR has the following components: a link reliability estimator, a queueing and transmission delay estimator, a queuing controller and a node forwarding metric incorporated with the dynamic velocity assignment policy. The proposed protocol RTTDR implements the modules for estimating queueing and transmission delay and packet delivery ratios using efficient methods. The packet delay is estimated at the node itself and the packet delivery ratio is estimated by the neighboring nodes. These parameters are updated on reception of a HELLO packet, the HELLO messages are periodically broadcast to update the estimation parameters. The overhead caused by the 1-hop and 2-hop updating are reduced by piggybacking the information in ACK, hence improving the energy efficiency. The notations used in this paper are given in Table 2. The protocol is based on the following parameters: (i) Link Reliability Estimation; (ii) Queueing and Transmission Delay Estimation; (iii) Node Forwarding Metric; and (iv) Queuing Controller

\subsection{Link Reliability Estimation}

The Packet Reception Ratio (PRR) of the link relaying node $x$ to $y$ is denoted by $p r r_{x y}$. It denotes the probability of successful delivery over the link. Window Mean Exponential Weighted Moving Average (WMEWMA) based link quality estimation is used for the proposed protocol. The window mean exponential weighted moving average estimation applies filtering on PRR, thus providing a metric that resists transient fluctuations of PRR, yet is responsive to major link quality changes. This parameter is updated by node $y$ at each window and inserted into the HELLO message packet for usage by node $x$ in the next window. Eq 1 shows the window mean exponential weighted moving average estimation of the link reliability, $r$ is the number of packets received, $m$ is the number of packets missed and $\beta \in[0,1]$ is the history control factor, which controls the effect of the previously estimated value on the new one, $\frac{r}{r+m}$ is the newly measured PRR value.

$$
p r r_{x y}=\beta \times p r r_{x y}+(1-\beta) \times \frac{r}{r+m}
$$

The PRR estimator is updated at the receiver side for each $w$ (window size) received packets, the computation complexity of this estimator is $O(1)$. The appropriate values for $\beta$ and $w$ for a stable window mean exponential weighted moving average are $w=30$ and $\beta=0.6|5|$.

\subsection{Queueing and Transmission Delay Estimation}

The nodal delay indicates the time spent to send a packet from node $x$ to its neighbor $y$, it is comprised of the queuing delay (delay ${ }_{Q}$ ), contention delay $\left(\right.$ delay $\left._{C}\right)$ and the transmission delay $\left(\right.$ delay $\left._{T}\right)$.

$$
\text { delay }_{n o d e}=\text { delay }_{Q}+\text { delay }_{C}+\text { delay }_{T}
$$

The queueing delay constitutes the time the packet is assigned to a queue for transmission and the time it starts being transmitted. During this time, the packet waits while other packets in the transmission queue are transmitted. Every node evaulates its queuing delay $d q_{x}$ for the various classes of queues used, i.e., Critical-Queue, Delay-Responsive-Queue, Reliability-ResponsiveQueue and Regular-Queue, each packet class has a different estimation of $d q_{x}$ for the queuing delay, i.e., $d q_{x}<$ packet.class $>$. Eq 3 shows the EWMA (Exponential Weighted Moving Average) update for queueing delay estimation, $d q$ is the current precise queue waiting time of the respetive packet and $\gamma \in[0,1]$ is the tunable weighting coefficient.

$$
\begin{aligned}
d q_{x}<\text { packet.class }>= & \gamma \times d q_{x}<\text { packet.class }> \\
& +(1-\gamma) \times d q
\end{aligned}
$$

The transmission delay represents the time that the first and last bits of the packet are transmitted. If $t_{s}$ is the time the packet is ready for transmission and becomes head of transmission queue, $t_{a c k}$ the time of the reception of acknowledgment, $B W$ the network bandwidth and size of the acknowledgment then, $t_{a c k}-$ sizeof $(A C K) / B W-t_{s}$ is the recently estimated delay. Eq 4 shows the EWMA (Exponential Weighted Moving Average) update for transmission delay estimation, which has the advantage of being simple and less resource demanding.

$$
d t_{x y}=\gamma \times d t_{x y}+(1-\gamma) \times\left(t_{a c k}-\operatorname{sizeof}(A C K) / B W-t_{s}\right)
$$

$d t_{x y}$ includes estimation of the time interval from the packet that becomes head of line of $x$ 's transmission queue until its reception at node $y$. This takes into account all delays due to contention, channel sensing, channel reservation (RTS/CTS) if any, depending on the medium access control (MAC) protocol, propagation, time slots etc. The computation complexity of both the estimators is $O(1)$. The delay information is further exchanged among two-hop neighbors.

\subsection{Node Forwarding Metric}

In the wireless sensor network, described by a graph $G=(N, L)$. If node $x$ can transmit a message directly to node $y$, the ordered pair is an element of $L$. We define for each node $x$ the set $N_{1}(x)$, which contains the nodes in the network $G$ that are one-hop i.e., direct neighbors of $x$.

$$
N_{1}(x)=\{y:(x ; y) \in E \text { and } y \neq x\}
$$




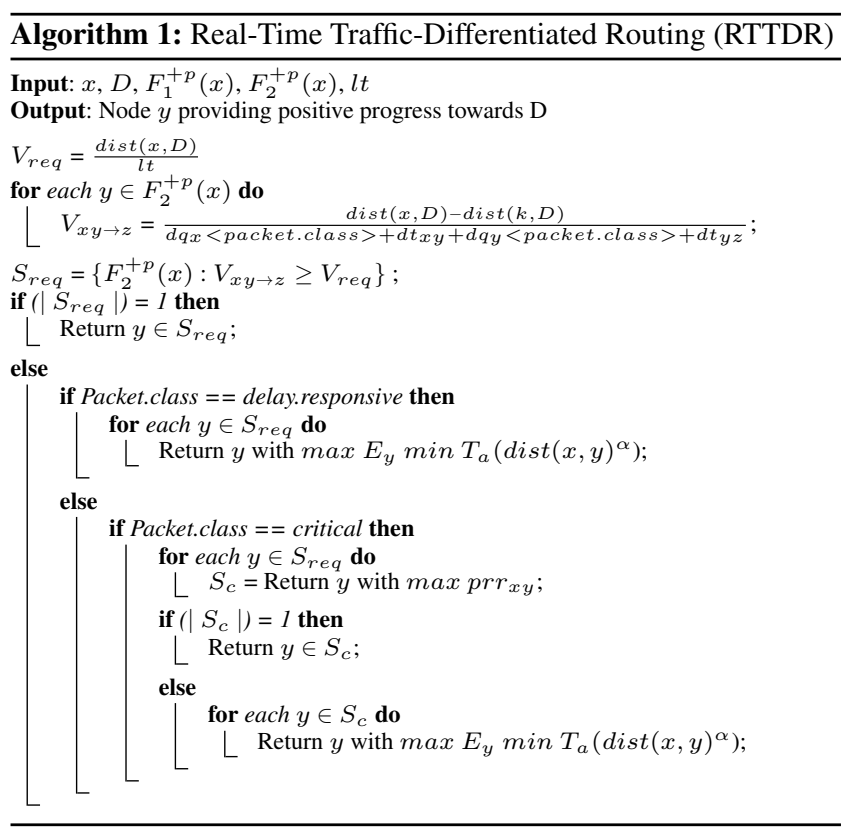

Likewise, the two-hop neighbors of $x$ is the set $N_{2}(x)$ i.e.,

$$
N_{2}(x)=\left\{z:(y ; z) \in E \text { and } y \in N_{1}(x), z \neq x\right\}
$$

The euclidean distance between a pair of nodes $x$ and $y$ is defined by $\operatorname{dist}(x, y)$. We define $F_{1}^{+p}(x)$ as the set of $x$ 's one-hop favorable forwarders providing positive progress towards the destination $D$. It consists of nodes that are closer to the destination than $x$, i.e.,

$$
F_{1}^{+p}(x)=\left\{y \in N_{1}(x): \operatorname{dist}(x, D)-\operatorname{dist}(y, D)>0\right\}
$$

$F_{2}^{+p}(x)$ is defined as the set of two-hop favorable forwarders i.e.,

$$
\begin{aligned}
F_{2}^{+p}(x)= & \left\{y \in F_{1}^{+p}(x), z \in N_{1}(y):\right. \\
& \operatorname{dist}(y, D)-\operatorname{dist}(z, D)>0\}
\end{aligned}
$$

We define two velocities; the required velocity $V_{r e q}$ and the velocity offered by the two-hop favorable forwarding pairs. In SPEED, the velocity provided by each of the forwarding nodes in $\left(F_{1}^{+p}(x)\right)$ is.

$$
V_{x y}=\frac{\operatorname{dist}(x, D)-\operatorname{dist}(y, D)}{d t_{x y}}
$$

As in THVR, by two-hop knowledge, node $x$ can calculate the velocity offered by each of the two-hop favorable forwarding pairs $\left(F_{1}^{+p}(x), F_{2}^{+p}(x)\right)$ as show in Eq 10 Furthemore, we include queuing delay at both the current $\left.\left(d q_{x}\right)\right)$ and the next hop $\left(d q_{y}\right)$ nodes, with the two-hop transmission delay $\left(d t_{x y}\right.$ and $\left.d t_{y z}\right)$, this distinguishes the proposed protocol from LOCALMOR.

$$
V_{x y \rightarrow z}=\frac{\operatorname{dist}(x, D)-\operatorname{dist}(z, D)}{d q_{x}<\text { packet.class }>+d t_{x y}+d q_{y}<\text { packet.class }>+d t_{y z}}
$$
relative to the progress made towards the destination [24] and the time remaining to the deadline, $l t$ (lag time). The lag time is the time remaining until the packet deadline expires. At each hop, the transmitter renews this parameter in the packet header i.e.,

$$
l t=l t_{p}-\left(t_{t x}-t_{r x}+\operatorname{sizeof}(\text { packet }) / B W\right)
$$

Where $l t$ is the time remaining to the deadline $\left(t_{r e q}\right), l t_{p}$ is the previous value of $l t,\left(t_{t x}-t_{r x}+\operatorname{sizeof}(\right.$ packet $\left.) / B W\right)$ accounts for the delay from reception of the packet until transmission. On reception of the packet the node $x$, uses $l t$ to calculate the required velocity $V_{r e q}$ for all nodes in $\left(F_{1}^{+p}(x), F_{2}^{+p}(x)\right)$ as show in Eq 12

$$
V_{\text {req }}=\frac{\operatorname{dist}(x, D)}{l t}
$$

The node pairs satisfying $V_{x y \rightarrow z} \geq V_{r e q}$ form the set of nodes $S_{r e q}$, if the packet class is delay.responsive then the node with the maximum residual energy and minimum transmission power cost is chosen from the set $S_{r e q}$. However, if the packet class is critical then the node with the highest packet reception ratio (PRR) is selected from $S_{r e q}$, but if more than one node has the same maximum PRR, a node with maximum power efficiency is picked.

The Real-Time Traffic-Differentiated Routing is shown in Algorithm 1, the computation complexity of this algorithm is $O\left(F_{2}^{+p}(x)\right)$. Our proposed protocol is different from LOCALMOR, as it considers two-hop neighborhood information that will provide enhanced foresight to the sender in identifying the node that can offer the required QoS and route the packets in real-time.

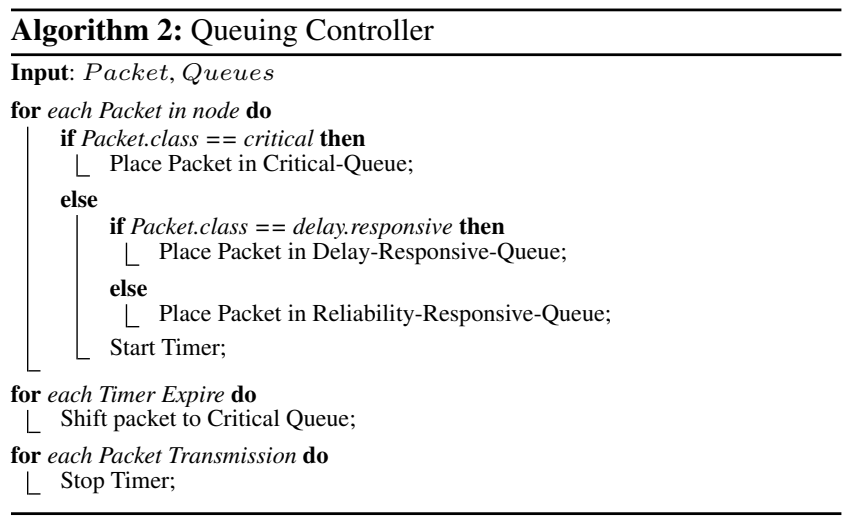

\subsection{Queuing Controller}

The queuing controller helps accomplish low delay when routing critical and delay-responsive packets, higher precedence should be given to these packets in channel contention than the normal packets (regular and reliability-responsive packets). Additionally, critical packets need higher priority than delay-responsive packets. This can be accomplished by implemented the queuing controller module as detailed in Algorithm 2 [11]. Three queues are used to send packets from the highest priority queue to the lowest one. The highest priority queue, Critical-Queue, is used by critical packets, the second highest priority queue, Delay-Responsive-Queue, is used by delay-responsive packets, and the least priority queue, Reliability-Responsive-Queue, is used by regular and reliabilityresponsive packets. The number of critical and delay-responsive packets is usually small, and there would be instances where their corresponding queues are vacant. Otherwise, lower priority traffic may be forever blocked by higher priority traffic. In this case, a timer for each packet is employed to move it to the highest priority queue. 


\section{PERFORMANCE EVALUATION}

To evaluate the proposed protocol, we carried out a simulation study using $n s-2$ [1]. In this study the proposed protocol (RTTDR) is compared with LOCALMOR, DARA and MMSPEED. The simulation configuration consists of 900 nodes located in a $1800 \mathrm{~m}^{2}$ area. Nodes are distributed following Poisson point process with a node density of 0.00027 node $/ \mathrm{m}^{2}$. The primary and secondary sink nodes are located in the region $(0,0)$ and $(1800,1800)$ while the source node is located in the center of the simulation area, equidistant from both the sinks. The source generated a CBR flow of 1 $\mathrm{kB} / \mathrm{second}$ with a packet size of 150 bytes. Critical and regular packets are used in the simulation for comparing our protocol with LOCALMOR, DARA and MMSPEED, while delay-sensitive and reliability-sensitive packets are used for comparing with LOCALMOR only. The deadline requirement was fixed in this simulation to $300 \mathrm{~ms}$ for all class of packets.

The MAC layer, link quality and energy consumption parameters are set as per TelosB ${ }^{3}$ (TPR2420) mote [2] with CC2420 radio as per LOCALMOR. Table 3 summarizes the simulation parameters. LOCALMOR, DARA and MMSPEED are QoS protocols and a comparison of PRR (Packet Reception Ratio), ECPP (Energy Consumed Per Packet i.e., the total energy expended divided by the number of packets effectively transmitted), packet average end-toend delay (mean of packet delay) and the network lifetime are obtained.

Table 3. Simulation Parameters.

\begin{tabular}{|l|c|}
\hline Simulation Parameters & Value \\
\hline Number of nodes & 900 \\
\hline Simulation Topology & 1800 x 1800 \\
\hline Traffic & CBR \\
\hline Critical Packet Rate & From 0 to 1 \\
\hline Regular Packet Rate & 1 - Critical Packet Rate \\
\hline Payload Size & 150 Bytes \\
\hline Transmission Power Range & $100 \mathrm{~m}$ \\
\hline Initial Battery Energy & 2.0 Joules \\
\hline Energy Consumed during Transmit & 0.0522 Joule \\
\hline Energy Consumed during Receive & 0.0591 Joule \\
\hline Energy Consumed during Sleep & 0.00006 Joule \\
\hline Energy Consumed during Idle & 0.000003 Joule \\
\hline MAC Layer & 802.11 with DCF \\
\hline Propagation Model & Free Space \\
\hline Hello Period & 5 seconds \\
\hline PRR - WMEWMA Window & 30 \\
\hline PRR - WMEWMA Weight Factor $(\alpha)$ & 0.6 \\
\hline Delay - EWMA Weight Factor $(\beta)$ & 0.6 \\
\hline
\end{tabular}

In the first set of simulations the critical packet rate was varied from 0.1 to 1 and the remaining rate to 1 represents regular packet rate. Fig. 1 and Fig. 2 illustrates the efficiency of the RTTDR algorithm in increasing the PRR with respect to regular and critical packets. RTTDR, LOCALMOR and DARA linearly increase their performance as a function of critical packet rate, while performance of MMSPEED is relatively stable. The high reliability of RTTDR, LOCALMOR and DARA is due to the use of efficient duplication toward different sinks, contrary to MMSPEED that uses a multipath single-sink strategy. This kind of duplication results in packet congestion either at the final sink or intermediate nodes.

In Fig. 2 the linear increase of the packet reception ratio for RTTDR, LOCALMOR and DARA with the increasing critical

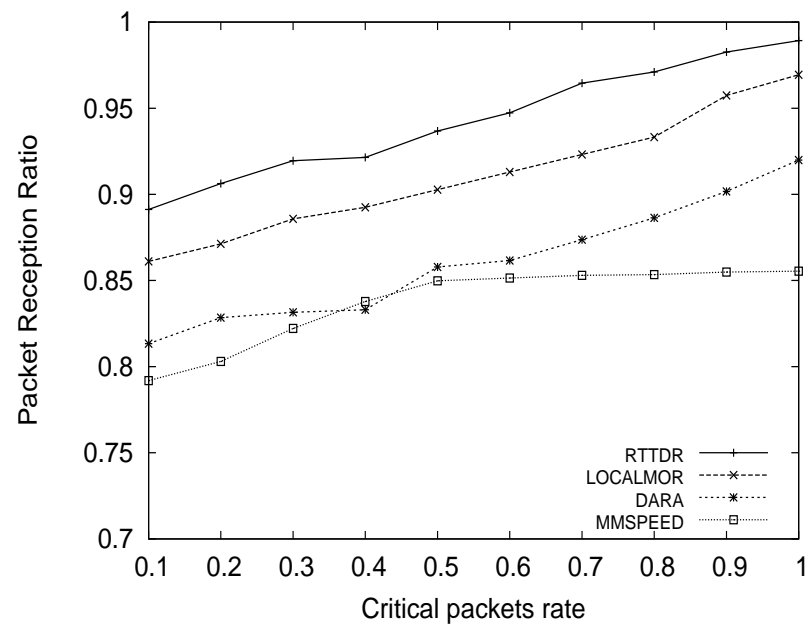

Fig. 1. Packet Reception Ratio - Regular Packets

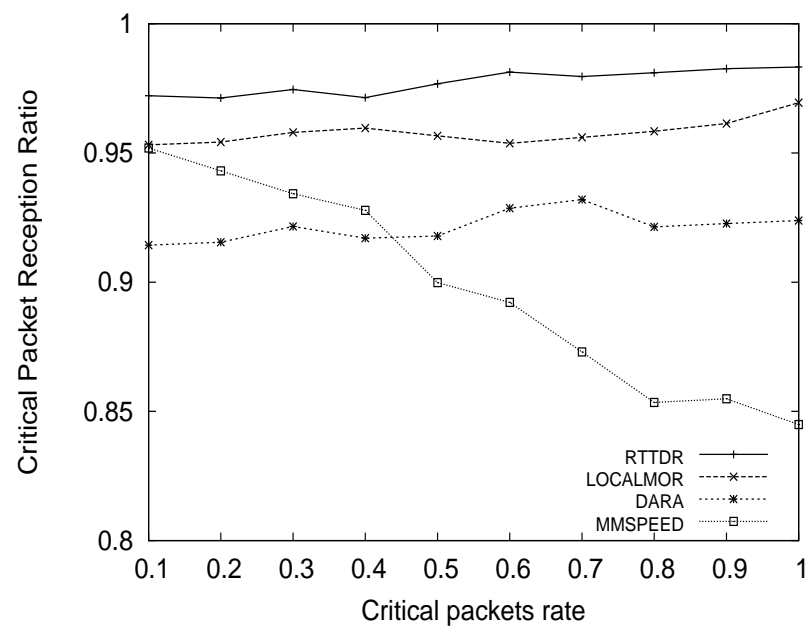

Fig. 2. Packet Reception Ratio - Critical Packets

packet rate can be explained by the subsequent increase of duplications (applied only to critical packets). This means, the larger the number of critical packets we have, the more the packets are duplicated, which subsequently increases their reception ratio. In RTTDR the two-hop based routing and dynamic velocity of the RTTDR algorithm is able to aggressively route more packets to the sink node, hence it is observed that RTTDR has higher PRR than the others in general.

Fig. 3 and Fig. 4 illustrate the packet end-to-end delay of regular packets and critical packets respectively, performance of LRTHR is better that the other protocols. LOCALMOR and DATA consider one-hop transmission delay and queuing waiting time, while RTTDR considers dynamic velocity, two-hop transmission delay and queuing waiting time hence the selected paths from source to sink will be shorter and aid in reducing the end-to-end delay. MMSPEED also considers queuing and transmission delays, but on the other hand, the use of multipath single-sink transmissions causes congestion and thus results in several retransmission of packets before successful reception, which explains the relatively higher de- 


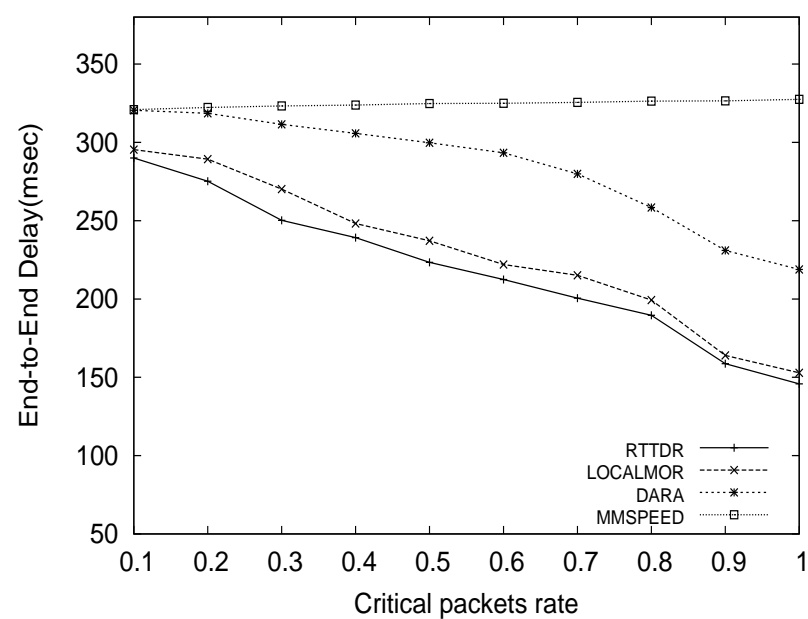

Fig. 3. End-to-End Delay - Regular Packets

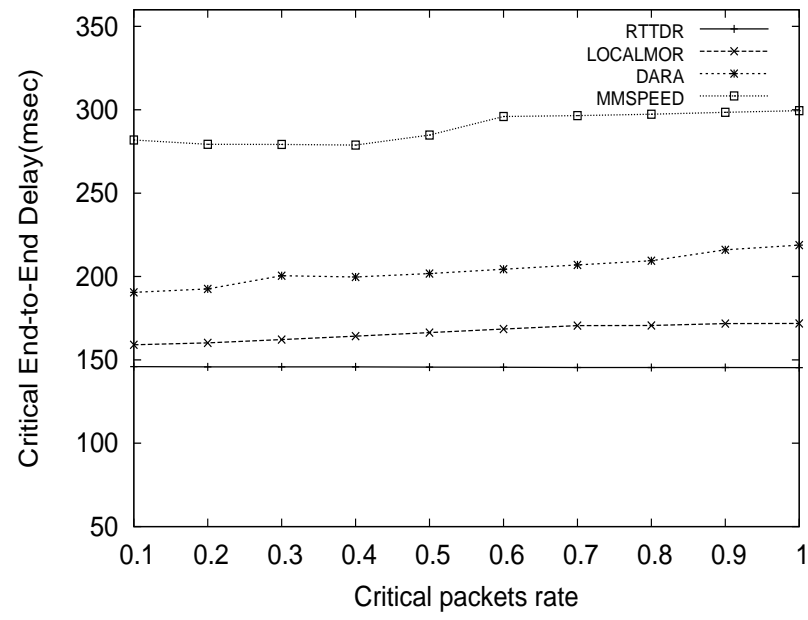

Fig. 4. End-to-End Delay - Critical Packets

lay. In Fig. 5 we notice a stable end-to-end delay for all protocols, which reflects the stability of the routes selected for critical packets that are obviously not affected by the rise in rate of critical packets. As depicted in Fig. 6 the energy consumption per packet (ECPP) successfully transmitted, ascend as the critical packet rate increases. The energy consumption has similar tendency in both RTTDR and LOCALMOR but DARA has a higher energy utilization. LOCALMOR ensures a trade-off between traffic related QoS metrics and energy, its ECPP smoothly increases as rates become higher. LOCALMOR balances the load only among nodes estimated to ensure delivery within the deadline and having the highest reliability. DARA performs poorly in terms of energy, as it does neither use any traffic balancing technique nor any probabilistic selection.

In RTTDR the two-hop based routing will ensure shorter paths between source and sink, by selecting links providing higher PRR on the route to the sink, the energy consumption of the forwarding nodes can be minimized, due to lower number of collisions and retransmissions and help in traffic balancing. Furthermore, in the pro-

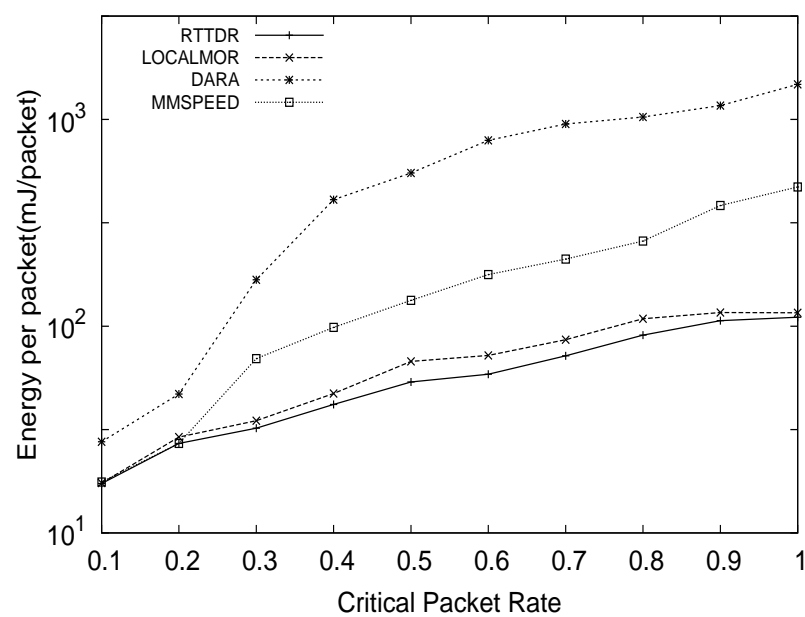

Fig. 5. ECPP vs Packet Rate

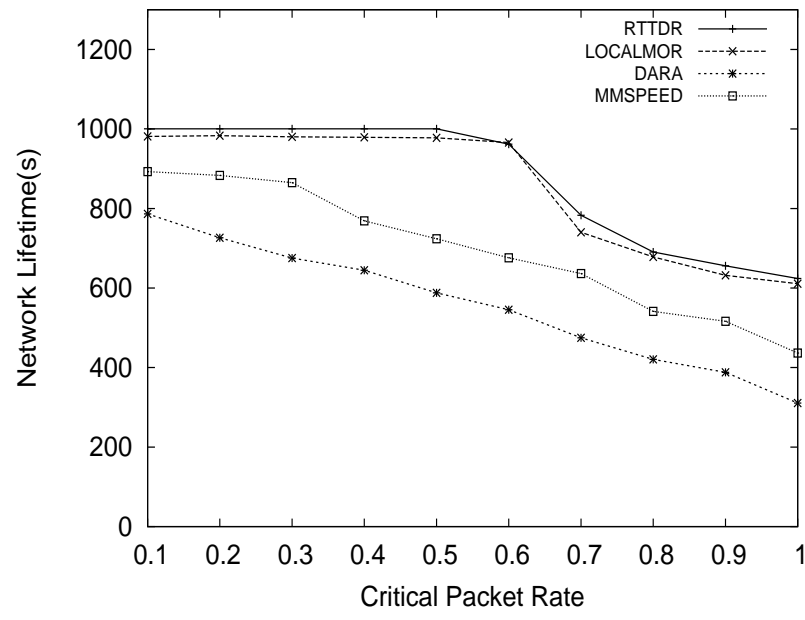

Fig. 6. Network Lifetime vs Packet Rate

posed protocol the link delay and packet delivery ratios are updated by piggybacking the information in ACK, this will help in reducing the number of feedback packets and hence reduce the total energy consumed. The impact of efficient energy utilization and traffic balancing on network lifetime is depicted in Fig. 6, RTTDR and LOCALMOR show good performance compared to DARA and MMSPEED.

Last, we study the performance of RTTDR and LOCALMOR with respect to delay-responsive and reliability-responsive traffic. The QoS traffic is varied in the same way as critical packets were varied in the earlier simulations, i.e., each QoS traffic varies from 0.1 to 1 . Fig. 7 and Fig. 8 examines the results, where the $\mathrm{x}$-axis represents the rate of QoS traffic. This comparison is important because we need to acertain the positive effect of two-hop delay incorporated in RTTDR over the one-hop delay used in the LOCALMOR. The delay-responsive traffic are routed through more delay efficient links, while reliability-responsive traffic, considers only reliable links. From Fig. 7 and Fig. 8 it is clear that the performance of RTTDR is better than LOCALMOR for delay-responsive 


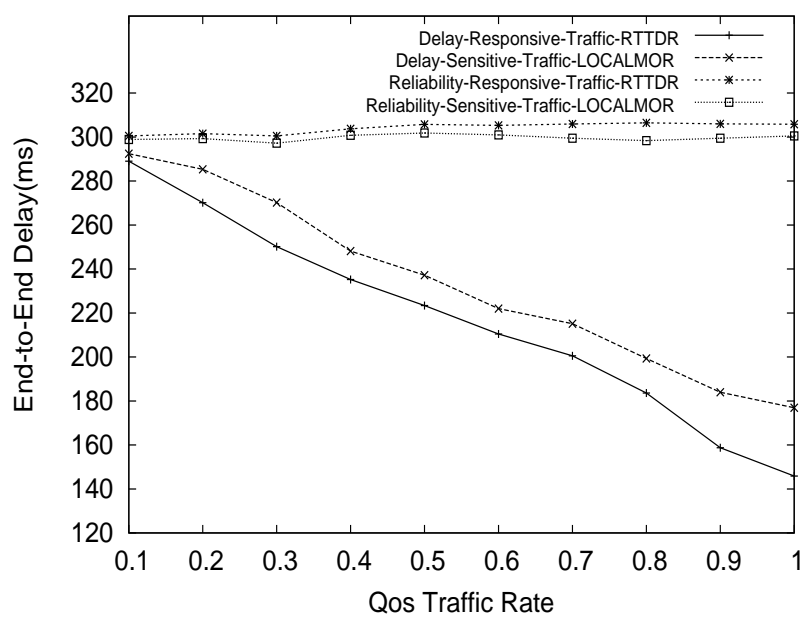

Fig. 7. RTTDR vs LOCALMOR - End-to-End delay

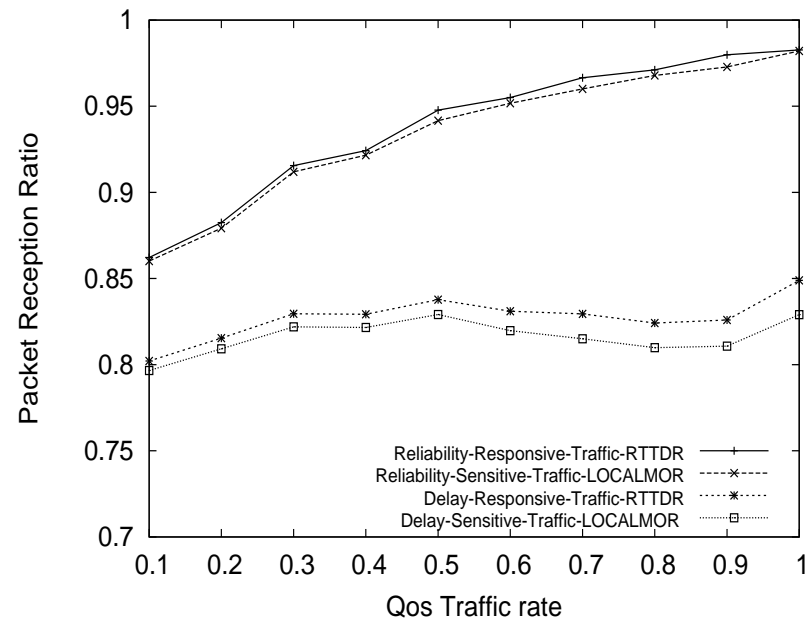

Fig. 8. RTTDR vs LOCALMOR - Packet Reception Ratio

traffic due to two-hop information and has similar performance for reliability-responsive traffic.

\section{CONCLUSIONS}

In this paper, we propose a Real-Time Traffic-Differentiated Routing quality of service (QoS) routing protocol for WSN, it provides a differentiation routing using different quality of service metrics. Data traffic has been sequenced into different classes according to the required QoS, where different routing metrics and techniques are used for each class. The consideration of two-hop neighborhood information and differentiation of both delay and reliability requirements distinguish the proposed protocol from the state-ofthe-art protocols. The protocol is able to augment real-time delivery by an able integration of multiqueue priority policy, link reliability, two-hop information and dynamic velocity. The protocol is able to reduce the DMR, end-to-end delay and improve the energy efficiency throughout the network. This makes the protocol suitable for WSN with varied traffic, such as medical and vehicular applications.

\section{REFERENCES}

\section{REFERENCES}

[1] NS-2, [Online]. Available: http://www.isi.edu/nsnam/ ns/

[2] Crossbow Motes, [Online]. Available: http://www.xbow. com

[3] A. Mahapatra, K. Anand, and D.P. Agrawal. QoS and Energy Aware Routing for Real-Time Traffic in Wireless Sensor Networks. in Computer Communications, 29(4):437-445, 2008.

[4] A. Sharif, V. Potdar and A.J.D Rathnayaka. Prioritizing Information for Achieving QoS Control in WSN. In Proc. IEEE International Conference on Advanced Information Networking and Applications, pages 835-842, 2010.

[5] A. Woo and Culler. Evaluation of Efficient Link Reliability Estimators for Low-Power Wireless Networks. Technical report, University of California, 2003.

[6] B. Karp and Kung. H. T. GPSR: Greedy Perimeter Stateless Routing for Wireless Networks. In Proc. 6th Annual International Conference on Mobile Computing and Networking (MobiCom), pages 243-254, 2000.

[7] C. Lu, B. M. Blum, T. F. Abdelzaher, J. A. Stankovic, and T. He. RAP: A Real-Time Communication Architecture for Large-Scale Wireless Sensor Networks. In Proc. IEEE RTAS, Sep. 2002.

[8] C.-p. Li, W.-j. Hsu, B. Krishnamachari, and A. Helmy. A Local Metric for Geographic Routing with Power Control in Wireless Networks. Proc. Second Ann. IEEE Conf. Sensor and Ad Hoc Comm and Networks (SECON), pages 229-239, Sept. 2005.

[9] C. S. Chen, Y. Li, and Y.-Q. Song. An Exploration of Geographic Routing with K-hop Based Searching in Wireless Sensor Networks. In Proc. CHINACOM, pages 376-381, 2008.

[10] D. Tran and H. Raghavendra. Routing with Congestion Awareness and Adaptivity in Mobile Ad Hoc Networks. In Proc. of IEEE WCNC, Mar. 2005.

[11] Djamel Djenouri and Ilangko Balasingham. TrafficDifferentiation-Based Modular QoS Localized Routing for Wireless Sensor Networks. in IEEE Transactions on Mobile Computing, 6(10):797-809, 2011.

[12] E. Felemban, C. G. Lee, and E. Ekici. MMSPEED: Multipath Multi-Speed Protocol for QoS Quarantee of Reliability and Timeliness in Wireless Sensor Network. in IEEE Transactions on Mobile Computing, 5(6):738-754, 2006.

[13] F. L. Lewis, D. J. Cook, S. K. Dasm and John Wiley. Wireless Sensor Networks. In Proc. Smart Environment Technologies, Protocols and Applications, New York, pages 1-18, 2004.

[14] G. Calinescu. Computing 2-hop Neighborhoods in Ad hoc Wireless Networks. In Proc. AdHocNow, pages 175-186, 2003.

[15] K. Seada, M. Zuniga, A. Helmy, and B. Krishnamachari. Energy Efficient Forwarding Strategies for Geographic Routing in Lossy Wireless Sensor Networks. In Proc. ACM SenSys, pages 108-121, 2004.

[16] K. Sohrabi, J. Pottie. Protocols for Self-organization of A Wireless Sensor Network. in IEEE Personal Communications, 7(5):16-27, 2000. 
[17] K. Zeng, K. Ren, W. Lou, and P. J. Moran. Energy Aware Efficient Geographic Routing in Lossy Wireless Sensor Networks with Environmental Energy Supply. in Wireless Networks, 15(1):39-51, 2009.

[18] K. Zeng, K. Ren, W. Lou, and P.J. Moran. Energy Aware Efficient Geographic Routing in Lossy Wireless Sensor Networks with Environmental Energy Supply. in Wireless Networks, 15(1):39-51, 2009.

[19] M. A. Spohn and J. J. Garcia-Luna-Aceves. Enhancing Broadcast Operations in Ad Hoc Networks with Two-Hop Connected Dominating Sets. Proc. IEEE MASS, pages 543545, 2004.

[20] M. Chen, V. Leung, S. Mao, Y. Xiao, and I. Chlamtac. Hybrid Geographical Routing for Flexible Energy-Delay Trade-Offs. in IEEE Transactions on Vehicular Technology, 58(9):49764988, 2009.

[21] M. Koulali, A. Kobbane, M. El Koutbi and M. Azizi. QDGRP : A hybrid QoS Distributed Genetic routing protocol for Wireless Sensor Networks. In Proc. International Conference on Multimedia Computing and Systems, pages 47-52, 2012.

[22] M. M. Or-Rashid, Md. Abdur Razzaque, M. M. Alam and C. S.Hong. Multi-Constrained QoS Geographic Routing for Heterogeneous Traffic in Sensor Networks. in IEICE Transactions on Communications, 91B(8):2589-2601, 2008.

[23] M.E Rusli, R. Harris and A. Punchihewa. Markov Chainbased analytical model of Opportunistic Routing protocol for wireless sensor networks. In Proc. TENCON IEEE Region 10 Conference, pages 257-262, 2010.

[24] O. Chipara, Z. He, G. Xing, Q. Chen, X. Wang, C. Lu, J. Stankovic, and T. Abdelzaher. Real-Time Power-Aware Routing in Sensor Network. In Proc. IWQoS, pages 83-92, Jun. 2006.

[25] Prosenjit Bose, Pat Morin, Ivan Stojmenovi, and Jorge Urrutia. Routing with Guaranteed Delivery in Ad hoc Wireless Networks. In Proc. of 3rd ACM Int. Workshop on Discrete Algorithms and Methods for Mobile Computing and Communications DIALM'99, pages 48-55, Aug. 1999.

[26] S. Ehsan, B. Hamdaoui and M. Guizani. Radio and Medium Access Contention Aware Routing for Lifetime Maximization in Multichannel Sensor Networks. in IEEE Transactions on Wireless Communication, 11(9):3058-3067, 2012.

[27] S. Wu and K.S. Candan. Power Aware Single and Multipath Geographic Routing in Sensor Networks. Proc. IEEE Intl Conf. Broadband Networks (BROADNETS 05), 5(7):974997, 2007.

[28] T. He, C. Huang, B.M. Blum, J.A. Stankovic, and T.F. Abdelzaher. Range-Free Localization and Its Impact on Large Scale Sensor Networks. in ACM Trans. Embedded Computer Systems, 4(4):877-906, 2000.

[29] T. L. Lim and M. Gurusamy. Energy Aware Geographical Routing and Topology Control to Improve Network Lifetime in Wireless Sensor Networks. In Proc. IEEE International Conference on Broadband Networks (BROADNETSO5), pages 829-831, 2005.

[30] T. Roosta, M. Menzo, and S. Sastry. Probabilistic Geographical Routing Protocol for Ad-Hoc and Sensor Networks. In Proc. Intl Workshop Wireless Ad-Hoc Networks (IWWAN), 2005.
[31] Tian He, John A. Stankovic, Chenyang Lu, and Tarek F. Abdelzaher. A Spatiotemporal Protocol for Wireless Sensor Network. in IEEE Transactions on Parallel and Distributed Systems, 16(10):995-1006, 2005.

[32] T.L. Lim and M. Gurusamy. Energy Aware Geographical Routing and Topology Control to Improve Network Lifetime in Wireless Sensor Networks. In Proc. IEEE Intl Conf. Broadband Networks (BROADNETS 05), pages 829-831, 2005.

[33] X. Wu, B.J. dAuriol, J. Cho, and S. Lee. Optimal Routing in Sensor Networks for In-Home Health Monitoring with Multi Factor Considerations. In Proc. Sixth Ann. IEEE Intl Conf. Pervasive Computing and Comm. (PERCOM 08), pages 720725, 2008

[34] Y. Li, C. S. Chen and Y.-Q. Song. Enhancing Real-Time Delivery In Wireless Sensor Networks With Two-Hop Information. in IEEE Transactions On Industrial Informatics, 5(2):113-122, 2009.

[35] Y. Sankarasubramaniam, B. Akan, and I. F. Akyildiz. ESRT:Event-to-Sink Reliable Transport in Wireless Sensor Networks. In Proc. of ACM Mobihoc, pages 177-188, 2003.

[36] Yunbo Wang, M.C Vuran and S. Goddard. Cross-Layer Analysis of the End-to-End Delay Distribution in Wireless Sensor Networks. in IEEE Transactions on Networking, 20(1):305$318,2012$. 